\title{
Claudia Lamberti
}

\section{Teorici di architettura ne "La letteratura artistica" di Julius von Schlosser}

Pubblicato in: "Roemische Historische Mitteilungen”, 47, 2005, p.503-513

Il presente contributo sui "Teorici di architettura nella Letteratura artistica di J. von Schlosser" ha lo scopo di effettuare una lettura particolare del testo di Schlosser, attraverso gli occhi dello studioso di storia dell'architettura alla ricerca di informazioni sugli scritti teorico-tecnici in questo campo. Compito di questo breve saggio è quindi il rilevare quali trattati di architettura Schlosser citi, ma anche, ove possibile, che opinione abbia dei loro autori. L'articolo elenca perciò tutti i passi della Letteratura artistica in cui si parla delle fonti in campo architettonico dal Medioevo al Settecento. Sebbene Schlosser nella sua attività storico-critica non si diffonda su tale disciplina, nè compia particolari studi sugli architetti, la lettura analitica del suo manuale per le fonti della storia dell'arte consente di evidenziarne la sensibilità verso i testi e gli autori più significativi nella storia dell'architettura. Questa sintesi può risultare un comodo ausilio per gli specialisti, per rintracciare immediatamente se ci siano nel testo di Schlosser notizie sulle edizioni di talune opere teoriche di architettura e volgersi quindi alla lettura del passo che le riguarda.

\section{La letteratura artistica e l'architettura.}

La letteratura artistica di Julius von Schlosser ${ }^{1}$ è un manuale delle fonti della storia dell'arte che si propone una "prima descrizione del materiale bibliografico" di elaborarlo criticamente, senza tuttavia, data la vastità dell'opera, scendere in profondità nei contenuti dei singoli trattati citati. Schlosser stesso sottolinea quanto ardua sia la sua impresa, e sa di poter prestare il fianco ad alcune critiche, per questo nella premessa riconosce che anche la descrizione del materiale bibliografico "potrà tuttavia aver bisogno di qualche indulgenza ${ }^{3}$ ".

\footnotetext{
${ }^{1}$ (Vienna 1866-1938)

${ }^{2}$ Cfr. J. V. SCHLOSSER, La letteratura artistica, Firenze 1996, 3.

${ }^{3}$ Cfr. SChlosser, 3.

C. Lamberti, Teorici di architettura ne La letteratura artistica di Julius von Schlosser, "Roemische Historische Mitteilungen", 47, 2005, p.503-513
} 
Riguardo alla pittura ed altre arti, il nostro autore mostra una profonda erudizione e una passione che lo porta a privilegiarle ed occuparsene più approfonditamente, mentre per l'architettura non si può negare che il lettore de La letteratura artistica percepisca la sensazione di un trattamento da arte "minore". Probabilmente, Schlosser avvertiva un certo distacco dalla materia e dagli aspetti sia matematico-teorici che tecnici, e non si è spinto in profondità nell' analisi dei pur numerosi trattati che la storia ci ha consegnato a testimonianza del fiorire di tale arte.

Va riconosciuto comunque a Schlosser il merito di aver minuziosamente seguito le sorti delle maggiori opere cinquecentesche sull'architettura, e di aver saputo scegliere, nell'arco dell'intera storia dell'architettura, i testi degli autori più importanti, pur non comprendendone fino in fondo la portata o pur non condividendo i lusinghieri giudizi esposti dai critici e dagli storici specializzati.

Questo articolo deve perciò limitarsi all'analisi delle menzioni degli scritti di teorici ed architetti, cercando di evidenziare il come e il perché delle presentazioni fatte da Schlosser, cui poco importava, per le finalità stesse de La letteratura artistica, l'espressione di un giudizio sulla progettazione o sull'architettura costruita dagli autori citati.

$\underline{\text { Sezioni de La letteratura artistica dedicate all' architettura. }}$

Le sezioni de La letteratura artistica in cui si affrontano scritti di architettura sono i libri I, II, IV, VI, IX.

$* * *$

\section{Libro I.}

All'inizio del Libro I (Il Medioevo), parte I (La letteratura artistica medievale. Sguardo generale), capitolo 2 (Nell'occidente latino), sezione A (Letteratura tecnica) Schlosser è lapidario: "L'antichità non ci ha lasciato nessun insegnamento di tecnica, se se ne eccettua Vitruvio"'. Nessun cenno ai contenuti del De architectura (fig. 1) segue questa sintetica affermazione, e solo 50 pagine dopo, nel Libro I (Il Medioevo), parte II (Sulla teoria dell'arte nel Medioevo), capitolo 1 (Il contributo dell'antichità), Schlosser tornerà

\footnotetext{
${ }^{4}$ SCHLOSSER , 25.

C. Lamberti, Teorici di architettura ne La letteratura artistica di Julius von Schlosser, "Roemische Historische Mitteilungen", 47, 2005, p.503-513
} 
a parlarne, si badi bene, considerandone gli aspetti normativi per i canoni e gli ordini, senza soffermarsi sulle considerazioni sul ruolo e la formazione dell'architetto o sulle numerose informazioni tecniche riportate nel trattato. Il Vitruvio di Schlosser, insomma, è soprattutto un esteta dell'architettura, la cui opera viene paragonata a quella compiuta dal retore Quintiliano nel campo della letteratura ${ }^{5}$.

Proseguendo la lettura del Libro I (Il Medioevo), parte I (La letteratura artistica medievale. Sguardo generale), capitolo 2 (Nell'occidente latino), sezione A (Letteratura tecnica), incontriamo la parte dedicata al Livre de portraiture o Album di Villard de Honnecourt, architetto francese del XIII secolo. Schlosser lo segnala come "tentativo di una specie di dottrina medievale delle proporzioni" e come "una delle fonti più importanti per la conoscenza dell'intima natura di quello stile che siamo soliti chiamare gotico e delle tradizioni che predominavano nei laboratori dell'alto Medio Evo", Non manca però di criticare la stilizzazione geometrica di figure ed architetture operata da Villard e di lamentarne la rinuncia "alla conoscenza anatomica e alla comprensione della realtà" evidenziando fin da queste pagine una personale predilezione per il Rinascimento ed il Barocco, quali momenti in cui emerge il gusto per il vero.

Successivamente Schlosser elenca un gruppetto di tarde pubblicazioni sui cantieri gotici e sottolinea come l'era successiva portò alla ribalta “in luogo del compasso gotico [...] le regole di Vitruvio e dei vitruviani" "." Ancora una volta, in un brevissimo cenno, Vitruvio appare come modello, ma non strettamente dal punto di vista tecnico. Il suo valore è per Schlosser quello di essere un'espressione dell'antichità, dell'estetica e dell'ordine classico cui guardarono gli architetti del Rinascimento.

\section{Libro II.}

Schlosser, nel libro II della Letteratura artistica (Il primo Rinascimento. L'eredità di Leonardo), dedica l'intera II parte ai teorici del primo Rinascimento.

Il primo capitolo è totalmente dedicato a Leon Battista Alberti, di cui si presenta il De re aedificatoria. Schlosser traccia un breve profilo dell'Alberti teorico, notandone la venerazione e al contempo la capacità critica nei confronti di Vitruvio, evidenziando

\footnotetext{
${ }^{5}$ SCHLOSSER, 68ss.

${ }^{6}$ SCHLOSSER, 33.

${ }^{7}$ SCHLOSSER, 34.

C. Lamberti, Teorici di architettura ne La letteratura artistica di Julius von Schlosser, "Roemische Historische Mitteilungen", 47, 2005, p.503-513
} 
come il suo trattato potesse rivolgersi ad un vasto pubblico di umanisti e non solo ai tecnici, sottolineando la moderna valenza nazionalista delle sue opere, volte ad evocare lo spirito degli antichi avi romani, ascendenti comuni di tutti gli italiani al momento divisi in molti stati dominati da poteri stranieri. Neanche una frase è dedicata da Schlosser all'Alberti architetto e alle sue realizzazioni, né, a dire il vero, sono spese molte parole per i contenuti del trattato, sorvolando anche sulla tematica del disegno e della prospettiva, che viene invece brevemente illustrata quando si passa a commentare il De pictura.

Il secondo capitolo è dedicato ai romantici del primo Rinascimento, così definiti da Schlosser perché gli parve di scorgere in loro una sensibilità, "un entusiasmo per l'antichità di natura romantica, proprio come quello delle posteriori generazioni di pittori $^{8}$ ". Tra questi scrittori vengono posti il Filarete e Francesco Colonna.

Del primo, Schlosser non loda le capacità letterarie, ritenendolo "assai inferiore all'Alberti", ma riconosce che egli ebbe "rapporti con la pratica più stretti" e cita il suo Ospedale milanese. Schlosser non perde occasione di indicare il trattato di Filarete come fonte di informazione sulle arti minori e la storia dell'iconografia, grazie alle minuziose descrizioni delle raccolte di gemme e delle allegorie contenute nel testo.

Colonna, presunto autore della Hypnerotomachia Poliphili, è citato poiché in quest'opera descrive un campo di antiche rovine che "rispecchia il fascino che quelle esercitavano e ancora esercitano sulla fantasia italiana",

Il terzo capitolo affronta i teorici puri del primo Rinascimento, ossia a detta di Schlosser "un gruppo di scrittori che cercano di determinare $\mathrm{i}$ fondamenti formali delle arti

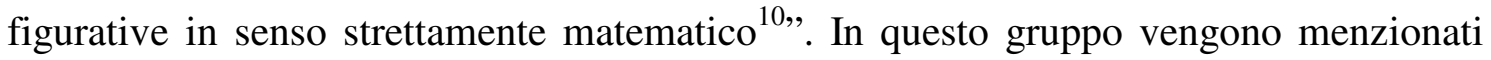
tutti coloro che in quel periodo si occuparono di proporzioni e prospettive, architetti, pittori, matematici: Francesco di Giorgio Martini, Piero della Francesca, Luca Pacioli, Giampaolo Lomazzo, Vincenzo Foppa, Bernardino Butinone, Bernardo Zenale, Bramante, Bramantino.

Francesco di Giorgio Martini è apprezzato come disegnatore e rilevatore di edifici antichi, poiché spesso le sue tavole assumono "il valore di una fonte diretta molto importante $^{11}$. . Grande rilevanza viene, giustamente, data da Schlosser alla sezione del

\footnotetext{
${ }^{8}$ SCHLOSSER, 133.

${ }^{9}$ SCHLOSSER , 135.

${ }^{10}$ SCHLOSSER, 138.

${ }^{11}$ SCHLOSSER, 139.

C. Lamberti, Teorici di architettura ne La letteratura artistica di Julius von Schlosser, "Roemische Historische Mitteilungen", 47, 2005, p.503-513
} 
Trattato di architettura civile e militare dedicata alle fortezze e alle macchine da guerra, campi in cui Francesco di Giorgio Martini primeggiò non solo come teorico ma come costruttore.

Attraverso la testimonianza di Lomazzo, Schlosser indica Butinone e Zenale come trattatisti d'architettura, attribuisce a Bramante la paternità di un disperso testo sulla "quadratura" del corpo umano ed equino e cita l'esistenza di un'opera sulla prospettiva scritta da Bartolomeo Suardi detto il Bramantino. Solo di quest'ultima Schlosser conosce con certezza i contenuti e si sofferma brevemente sulle tre maniere di costruzioni prospettiche ivi descritte.

\section{Libro IV.}

Nella III parte (Continuazione degli studi su Vitruvio) del libro IV (La teoria dell'arte nella prima metà del secolo XVI), Schlosser traccia una breve storia della trasmissione del De architectura e segnala la prima traduzione stampata del trattato vitruviano, quella del 1521 ad opera di Cesare Cesariano. In seguito loda la versione di Daniele Barbaro del 1556 che "oscurò tutte le precedenti ${ }^{12}$ ". Il grande interesse cinquecentesco verso il celebre trattatista latino portò ad un fiorire di emulatori più o meno competenti e capaci, e Schlosser cita, tra gli altri, gli umanisti ed i nobili italiani che si vollero cimentare nel campo dell'architettura: Francesco Maria Grapaldi, autore del De partibus aedium libri duo, "un vero lessico di tutte le espressioni che si riferiscono alla casa nell'antichità nel senso più vasto, pensato e condotto in modo rigorosamente filologico ${ }^{13,}$; Luigi Cornaro, che scrisse un Trattato dell'architettura in cui fornì giudizi spregiudicati per l'epoca, sostenendo che un edificio può essere bello e comodo "senza antichità", senza essere in qualche stile; i membri dell'Accademia delle virtù a Roma (Giangiorgio Trissino, Bernardino Maffei, Filandro, Claudio Tolomei), che avevano progettato un'edizione filologica del De architectura, un Lexicon vitruvianum, un'opera che confrontasse le regole di Vitruvio coi resti antichi ancora esistenti ed infine un Corpus di rilievi (programma irrealizzato se non in scritti apparsi in diverse occasioni lungo i secoli XVI e XVII).

\footnotetext{
${ }^{12}$ SCHLOSSER, 252.

${ }^{13}$ SCHLOSSER, 253.

C. Lamberti, Teorici di architettura ne La letteratura artistica di Julius von Schlosser, "Roemische Historische Mitteilungen", 47, 2005, p.503-513
} 
Schlosser passa poi a ricordare che "non erano mai cessate le opere degli architetti veri e propri" ${ }^{\text {"14 }}$, menzionando ad esempio uno scritto di Francesco Sansovino, figlio di Jacopo, dedicato alle relazioni tra corpo umano e pianta o sezione architettonica, tema legato alla teoria vitruviana della proporzione e sviluppatosi molto nel Rinascimento sotto l'impulso del neoplatonismo.

Schlosser segnala inoltre la possibile esistenza di un trattato di Baldassarre Peruzzi, non conservato, ma citato da altri autori. I suoi contenuti furono probabilmente esposti dall'allievo Sebastiano Serlio nella sua opera.

Nel complesso, in questa sezione de La letteratura artistica, Schlosser sceglie di privilegiare l'esposizione di studi vitruviani a carattere filologico, filosofico o dilettantistico, per riservare al VI libro una parte esplicitamente dedicata alle opere dei grandi architetti del XVI secolo.

\section{$\underline{\text { Libro VI. }}$}

Il libro VI (La letteratura artistica nel periodo del manierismo), parte III (I maestri dell'architettura), appare al lettore come il momento de La letteratura artistica in cui Schlosser parla di storia dell' architettura con maggiore diffusione e trasporto, rivelando una predilezione per alcuni degli autori di questo periodo: Serlio, Palladio, Scamozzi (fig. 2). Alle loro opere il critico austriaco dedica una spazio molto maggiore che ad altri architetti, sia perché si tratta di personalità indiscutibilmente eccelse, sia perché le loro pubblicazioni ebbero lunghe vicissitudini. All'interno del quartetto dei grandi, Vignola riceve invece giudizi più tiepidi da parte di Schlosser.

Riguardo l'opera di Sebastiano Serlio (fig. 3), Schlosser non esita a definirla (per una quantità di volte nel corso del testo!) importante, di "non comune valore"," "uno strepitoso successo ${ }^{16,}$, "il primo tentativo di un sistema architettonico nel senso dell' età nuova e dei postulati dopo L. B. Alberti ${ }^{17}$,"

Gli scritti di Serlio generarono stuoli di seguaci ed imitatori, di cui molti dilettanti, e tra questi Schlosser pone Jacopo Barozzi da Vignola, definendolo "il vero pedante

\footnotetext{
${ }^{14}$ SCHLOSSER, 255.

${ }^{15}$ SCHLOSSER , 407.

${ }^{16}$ SCHLOSSER , 409.

${ }^{17}$ SCHLOSSER, 409.

C. Lamberti, Teorici di architettura ne La letteratura artistica di Julius von Schlosser, "Roemische Historische Mitteilungen", 47, 2005, p.503-513
} 
dell'architettura $^{18 \%}$. Pur riconoscendogli il merito di aver costruito alcuni dei più importanti monumenti dell'epoca e di aver ottenuto un clamoroso successo editoriale con le Regole delli cinque ordini dell'architettura (fig. 4-5), Schlosser non nutre una grande simpatia verso l'opera del Vignola, parlando del trattato come di un testo "chiaro, sobrio, obiettivo, ben disposto, ma anche fatto di sole formule e privo di vita fino all'incredibile ${ }^{19,}$.

Su Palladio, Schlosser si pronuncia dicendo che "il più celebre trattatista d'architettura di tutto il gruppo è anche il maggiore come $\operatorname{artista}^{20 "}$ e considerando la sua opera “perfetta nell'esposizione ${ }^{21}$ " e molto "superiore per ricchezza e per importanza ${ }^{22,}$ rispetto a quella del Vignola.

Vincenzo Scamozzi, autore dell'Idea dell'architettura universale, è studiato da Schlosser con interesse, sia perché nel suo libro offre "un sommario storico dei maggiori architetti e scrittori di architettura $^{23}$ ", sia perché la sua opera unisce istruzione pratica con caratteri letterari e didascalici. Tuttavia gli si rimprovera una "erudizione pesante e non sempre digerita ${ }^{24, "}$ ed una "ampiezza quasi insopportabile ${ }^{25}$ " nell' esporre le basi teoriche dell'architettura, notando infine una caduta di stile nell'VIII e ultimo libro dei dieci previsti "redatto senza alcun ordine e in fretta, con mano sempre più debole ${ }^{26,}$.

$$
* * *
$$

\section{Libro IX.}

Nel libro IX (La teoria artistica dei secoli XVII e XVIII), parte I (La teoria artistica dei secoli XVII e XVIII (sommario)) Schlosser dedica un paio di pagine alla trattatistica architettonica. L'introduzione recita: "La letteratura propriamente tecnica di questo periodo non si può in alcun modo paragonare a quella del periodo precedente. Come era

\footnotetext{
${ }^{18}$ SCHLOSSER , 409.

${ }^{19}$ SCHLOSSER , 411.

${ }^{20}$ SCHLOSSER , 414.

${ }^{21}$ SCHLOSSER, 415.

${ }^{22}$ SCHLOSSER, 414.

${ }^{23}$ SCHLOSSER , 416.

${ }^{24}$ SCHLOSSER, 416.

${ }^{25}$ SCHLOSSER , 416.

${ }^{26}$ SCHLOSSER, 417.

C. Lamberti, Teorici di architettura ne La letteratura artistica di Julius von Schlosser, "Roemische Historische Mitteilungen", 47, 2005, p.503-513
} 
da attendersi continua nella stessa misura l'attività nel campo della teoria architettonica, specialmente dell'architettura civile; ma tutti questi trattati (del Viola, del Barca, del Capra) scompaiono nell'ombra di fronte ai grandi sistemi del Cinquecento che continuano a godere di piena considerazione e sono sempre ristampati e rimaneggiati; gli altri superano raramente i confini della loro patria. Un po' diversamente accade di un altro ramo di questa letteratura, che ha strettissimi rapporti coll'architettura e anche colla scultura e pittura, attraverso le sempre più rigogliose creazioni del teatro e dell'opera: la letteratura dei prospettici ${ }^{27,}$. Schlosser giudica il Seicento come un secolo dell'illusionismo architettonico, più che dell'architettura e preferisce perciò dedicare più spazio al Perspectiva pictorum et architectorum di Andrea Pozzo (1693) che ai trattati “classici”, dei quali comunque è menzionata una gran quantità nella bibliografia. Si noti che anche agli scritti di grandi autori, quali Borromini e Guarini, Schlosser non riserva che una menzione bibliografica, giustificando questo trattamento con la scarsa popolarità che tali opere ebbero presso i contemporanei.

Tra i testi tecnici del Seicento viene citato quello del senese Teofilo Gallaccini, un saggio sugli Errori degli architetti, rimasto inedito fino al 1767, quando Carlo Lodoli ne curò la pubblicazione. Esso “è un'opera che tratta degli errori degli architetti specialmente nella costruzione e nella tecnica, ma considera anche vere e presunte mancanze contro la forma ${ }^{28,}$.

Schlosser tornerà a parlare del ruolo di Lodoli come patrono e cultore della teoria dell'architettura nel Libro IX (La teoria artistica dei secoli XVII e XVIII), Parte V (La teoria artistica italiana del secolo XVIII). Egli non scrisse nulla, ma le sue idee furono trasmesse dagli allievi, che lo definirono ora un grammatico ora un filosofo dell'architettura e ne diffusero la moderna intuizione, molto apprezzata da Schlosser, secondo cui il fine di un'opera d'arte (ad esempio una gondola) è pienamente raggiunto quando "ogni pezzo corrisponde perfettamente alla sua funzione e suscita così anche l'impressione della bellezza artistica ${ }^{29, "}$

Tra gli architetti italiani del Settecento, Schlosser sceglie di far cenno a Ferdinando Galli Bibiena (fig. 6) e a Francesco Milizia (fig. 7). Di quest'ultimo si dice che "non riscosse certo molti allori come architetto, ma fu uno dei più autorevoli, più noti e più

\footnotetext{
${ }^{27}$ SCHLOSSER , 620.

${ }^{28}$ SCHLOSSER , 620-621.

${ }^{29}$ SCHLOSSER , 667.

C. Lamberti, Teorici di architettura ne La letteratura artistica di Julius von Schlosser, "Roemische Historische Mitteilungen", 47, 2005, p.503-513
} 
temuti critici d'arte". ${ }^{30}$ Milizia emerge nella descrizione di Schlosser come un purista, feroce avversario del barocco e anticipatore del neoclassicismo napoleonico ed ottocentesco, di cui La letteratura artistica non arriva ad occuparsi, terminando in questa sezione l'arco cronologico che essa copre. Schlosser conclude la trattazione della storia delle fonti architettoniche settecentesche inserendo alla fine di questa parte una vasta bibliografia, suddivisa per paese.

$$
* * *
$$

Bibliografia.

J. v. SCHLOSSER, La letteratura artistica, Firenze 1996.

$$
* * *
$$

$\underline{\text { Referenze fotografiche. }}$

Fondo storico della Biblioteca di Ingegneria dell’Università di Pisa.

\footnotetext{
${ }^{30}$ SCHLOSSER , 667.

C. Lamberti, Teorici di architettura ne La letteratura artistica di Julius von Schlosser, "Roemische Historische Mitteilungen", 47, 2005, p.503-513
} 